\title{
The perception of mobile appliances users on the changes brought to their lifestyle
}

\author{
Alexandru Zalana, Kantor Janos, Silvia Pop, Mariana Pacurar
}

UMFST „G.E. Palade“, Tg. Mures, Romania

\begin{abstract}
Dento-maxilares anomalies can cause severe three-dimensional disorders of the dental arches and implicitly of dental occlusion, interfering with the harmonious development of the physiological dentition, so is necessary to treat the anomalies as soon is possible. The earlier the age of intervention, the higher the success of an orthodontic treatment, having the possibility of guiding the remaining growth, of controlling the dental eruption and permutation, of correcting bad habits by remouvable or fixed appliances.

Aim. The authors have done an evaluation of the oral hygiene practices of patients undergoing mobile orthodontic therapy and the impact on their diet and lifestyle in general.

Material and method. A questionnaire with 14 questions has been distributed to a number of 63 patients undergoing the active stage of the orthodontic treatment with mobile appliances at the school medical centre in Bucharest, School no. 5. Children aged up to 10 years old have been helped by their parents to fill out this survey, and older children filled out the survey on their own.

Results. Most of the respondents have understood the importance of the dental appliance hygiene and teeth hygiene, and then almost in equal shares they have understood also, the importance of diet and of auxiliary means of hygiene. Majority of children (high percentage of the patients $80 \%$ ), have indicated that the orthodontic treatment will benefit them an improved esthetic aspect and healthy teeth. They also noticed the importance of the dental appliance hygiene and teeth hygiene, and then have understood, the importance of diet and of auxiliary means of hygiene $(89 \%)$.

Conclusion. There is an obvious tendency of increased desire for an orthodontic therapy within the analyzed population, most of the patients being aware of the role and need to wear an orthodontic appliance
\end{abstract}

Keywords: orthodontic treatment, bad habits, oral hygiene, appliances

\section{INTRODUCTION}

Orthodontic treatment helps to restore a functional occlusion, improving the aesthetics and functionality of the dental-facial complex and implicitly the quality of life. A correct alignment of the teeth, correcting some dental or skeletal anomalies, improves the possibilities of dental hygiene, reducing the risk of periodontal impairment. The earlier the age of intervention, the higher the success of an orthodontic treatment, having the possibility of guiding the remaining growth, of controlling the dental eruption and permutation, of correcting bad habits, the results obtained being better and stable ones $(1,2)$. Dento-maxilares anomalies can cause severe threedimensional disorders of the dental arches and implicitly of dental occlusion, interfering with the harmonious development of the physiological dentition, so is necessary to treat the anomalies as soon is possible.
The mobile appliances indicated for an early age have multiple advantages, but also a great disadvantage regarding their use as it depends of the patient's compliance that may be quite reduced especially in small schoolchildren.

There are several studies published that have attempted to establish the correlation between the indicator of dental caries in mixed dentition and the risk of maxillary dental anomalies, as well as the way in which intra-arch and inter-arch modifications occur. It is very important to analise the level of stress during the orthodontic treatment and the compliance of patiens which wearing remouvable appliances (3).

\section{PURPOSE}

Assessment of the oral hygiene practices of patients undergoing mobile orthodontic therapy and the impact on their diet and lifestyle in general. 


\section{MATERIAL AND METHOD}

A survey with 14 questions has been distributed to a number of 63 patients undergoing the active stage of the orthodontic treatment with mobile appliances at the school medical centre in Bucharest, School no. 5. Children aged up to 10 years old have been helped by their parents to fill out this survey, and older children filled out the survey on their own. The survey is a personal creation, with multiple answer possibilities, concisely formulated and easy to understand by each patient.

The children have filled out the survey in the waiting room, before entering for their check-up, at intervals of 1 month, 3 months and 6 months from the time they received their appliance. Filling out the survey took them about 10 minutes, they then returned it, and the answers were statistically processed in the Excel program.

The survey is anonymous and includes questions structured in three parts. The first three are general questions, containing personal data. According to their age the patients have been divided in three groups: 6-11 years old, 11-14 years old and 14-16 years old. We have taken under consideration also the gender of the patients.

The next 6 questions (4-9) are related to the oral hygiene after receiving an orthodontic appliance and how it suffered some changes (duration, type of tooth brushing).

The last 4 questions are related to the patient's compliance to use the orthodontic appliance, containing also psychological aspects: esthetic expectations related to the appliance, the classmates' perception of the patients after applying the orthodontic appliances, the patients' perception on bad habits.

The survey on the perception of patients with mobile orthodontic appliances is the following:
1. Your age
a. 6-11 years old
b. 11-14 years old
c. 14-16 years old

\section{Your gender}

a. Female

b. Male

3. How long did you start the orthodontic treatment?
a. 1 month
b. 3 months
c. 6 months

4. Which were the additional indications received after the application of the orthodontic appliance:

a. appliance hygiene and teeth hygiene

b. diet hygiene (reducing sugar intake)

c. use of additional means of hygiene (dental floss, fluoride mouthwash solutions)
5. What type Manual
b. Electric
c. Both

6. How has the application of the dental appliance influenced the hygiene possibilities?

a. Increase of tooth brushing time

b. Increase of tooth brushing frequency

c. It has not influenced me

7. How many times do you brush your teeth?

a. Once a day

b. Twice a day

c. Three times a day or more

8. How long it takes to brush your teeth?

a. 1-2 minutes

b. 3-5 minutes

c. 5-10 minutes

9. Do you use mouthwash?

a. Once a day

b. Twice a day

c. Never

10. How fast did you get used to the orthodontic appliance?
a. very fast
b. relatively fast
c. very hard

11. Do you consider important to use an appliance?

a. very important

b. less important

c. I don't know, my parents brought me

12. What bad habits do you have?

a. finger sucking

b. onychophagia (nail biting)

c. mouth breathing

d. infantile deglutition (tongue thrust swallowing) 
13. What is the impact of the orthodontic appliance on school activity?

a. I relate better with my classmates

b. I am part of the envied group

c. classmates avoid me (they make jokes)

14. Which do you think is the major benefit of using an orthodontic appliance?

a. a more beautiful smile, healthier teeth

b. improves chewing

c. increase of self-esteem

d. giving up bad habits

\section{RESULTS}

The distribution by age groups is relatively homogenous, the highest number of patients are in the interval of 6-11 years old (64\%), the stage of mixed dentition, optimum for the changes induced by orthodontic appliances. The lowest number of patients is noticed in the interval of 14-16 years old $(0,9 \%)$ (figure 1$)$.

\section{Your age \\ a. 6-11 years old \\ b. 11-14 years old \\ c. 14-16 years old}

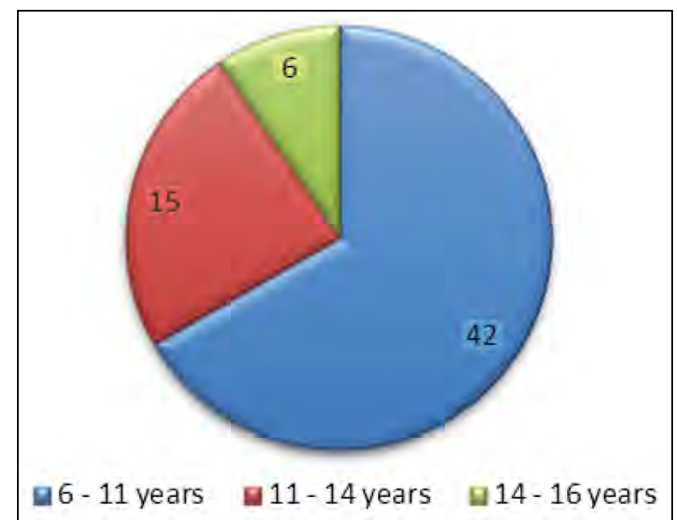

FIGURE 1. Patients' age

\section{Your gender}

a. Female

b. Male

It is observed a higher share of $61 \%$ in the female gender, compared to $39 \%$ in the male gender (figure 2 ). In general, girls are more receptive regarding the facial esthetic aspect, including the dental aspect, and consequently the degree in which they address this aspect is higher, compared to the male gender.

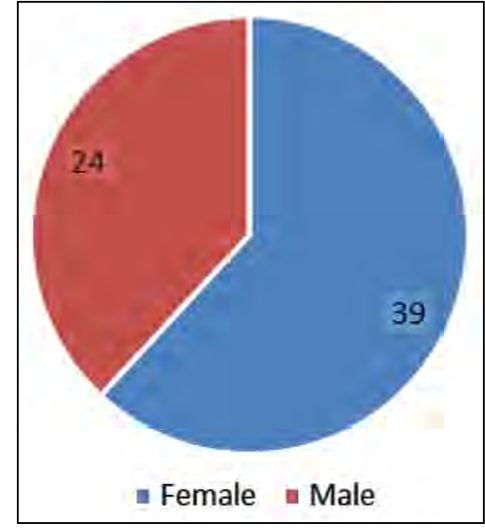

FIGURE 2. Distribution by gender

3. How long did you start the orthodontic treatment?

a. 1 month

b. 3 months

c. 6 months

Most of the respondents, $61 \%$, are newly beneficiaries of appliances, and by filling out this survey have the advantage of relatively fresh information/training and perception in their memory.

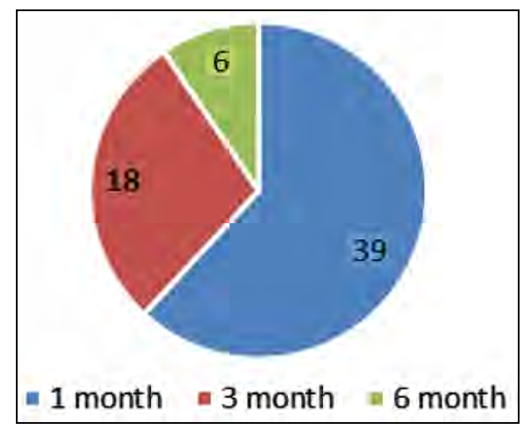

FIGURE 3. Initiating orthodontic treatment

4. Which were the additional indications received after the application of the orthodontic appliance:

a. appliance hygiene and teeth hygiene

b. diet hygiene (reducing sugar intake)

c. use of additional means of hygiene (dental floss, fluoride mouthwash solutions)

In this question of the questionnaire, most of the respondents have understood the importance of the dental appliance hygiene and teeth hygiene, and then almost in equal shares they have understood also the importance of diet and of auxiliary means of hygiene $89 \%$ (figure 4 ). 


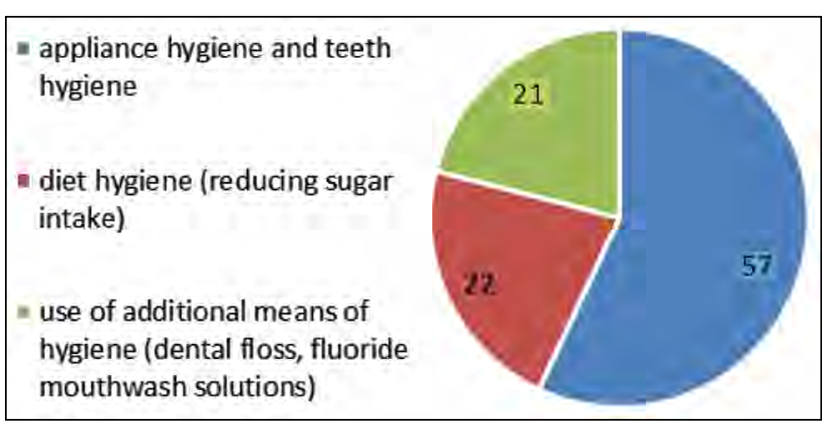

FIGURE 4. Indications received at the beginning of the treatment
5. What type of toothbrush are you using?
a. Manual
b. Electric
c. Both

The manual brush is the most used means of oral hygiene, while $23.44 \%$ use the electric brush, and $17.97 \%$ use alternatively both types of brushes (figure 5).

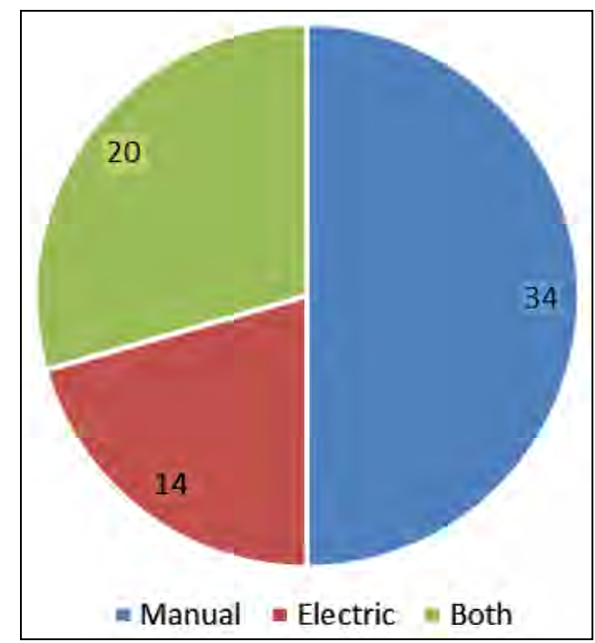

FIGURE 5. Type of toothbrush used

6. How has the application of the dental appliance influenced the hygiene possibilities?

a. Increase of tooth brushing time

b. Increase of tooth brushing frequency

c. It has not influenced me

Only $5 \%$ of the patients have not noticed changes regarding their oral hygiene habits (changes in frequency and duration). $53 \%$ of the patients have noticed increases in the tooth brushing frequency and duration, $30 \%$ have noticed increases only in the duration of tooth brushing, and $12 \%$ have noticed increases in the tooth brushing frequency (figure 6).

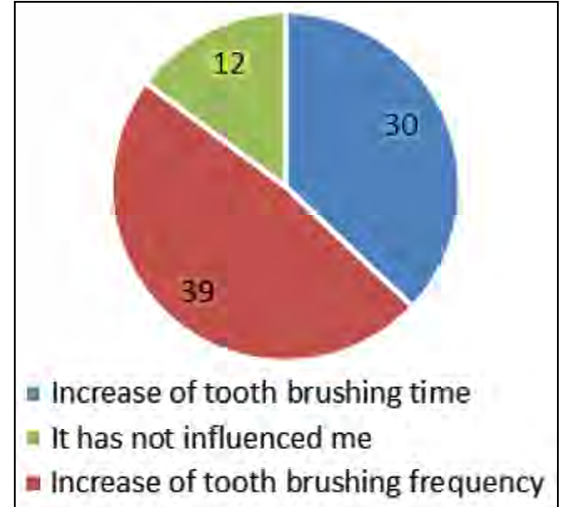

FIGURE 6. Correlations between the orthodontic appliance and dental hygiene

\section{How many times do you brush your teeth?}

a. Once a day

b. Twice a day

c. Three times a day or more

Most patients practice regular tooth brushing, twice or several times a day $(45 \%$, respectively $48 \%)$, a considerable higher percentage is represented by the patients who practice a tooth brushing with a duration of 3-5 minutes (57\%) (figure 7).

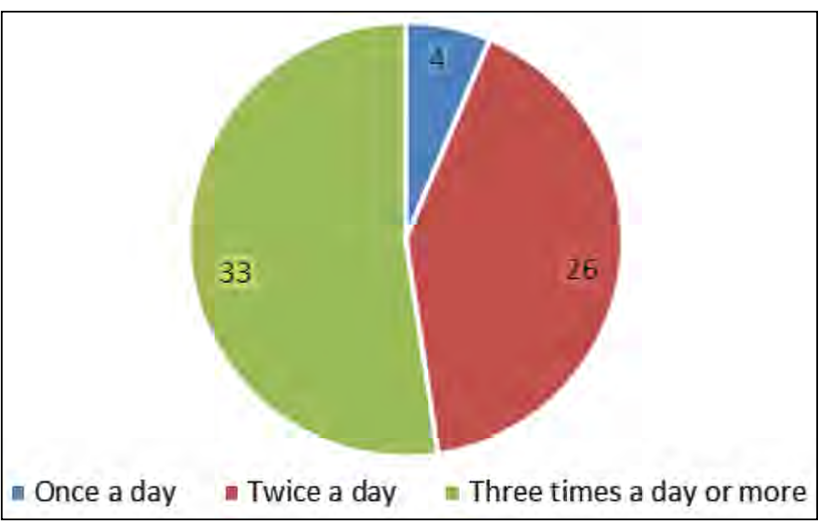

FIGURE 7. Tooth brushing frequency

Analyzing the female gender patients' rate of answers, compared to the male gender patients, we determined that female patients perform tooth brushing three or more times a day (61\%), compared to male patients who perform tooth brushing predominantly twice a day $(55 \%)(\mathrm{p}=$ 0.0044) (figure 8).
8. How long it takes to brush your teeth?
a. 1-2 minutes
b. 3-5 minutes
c. 5-10 minutes 


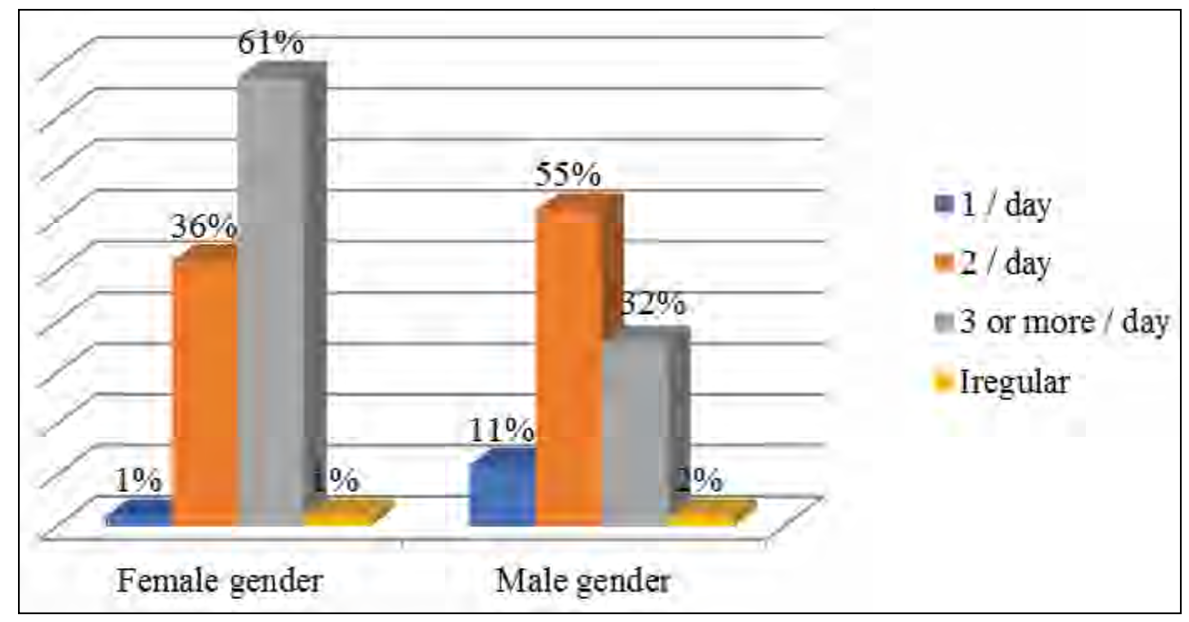

FIGURE 8. Correlations between frequency of tooth brushing and gender

Most of the patients (55\%) practice tooth brushing for an average duration of 3-5 minutes (figure 9).

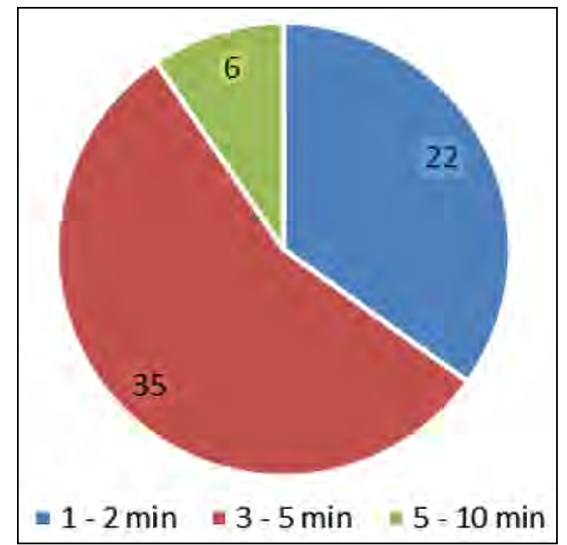

FIGURE 9. Duration of tooth brushing

In order to correlate the patients age with the duration of tooth brushing, we have applied a Kruskal Wallis nonparametric test, which shows that the median age in children with 1 tooth brushing per day for 3-5 minutes was the highest, $p$ $=0.007$ (fig. 10), compared to 1 tooth brushing per day for 5-10 minutes (figure 11).

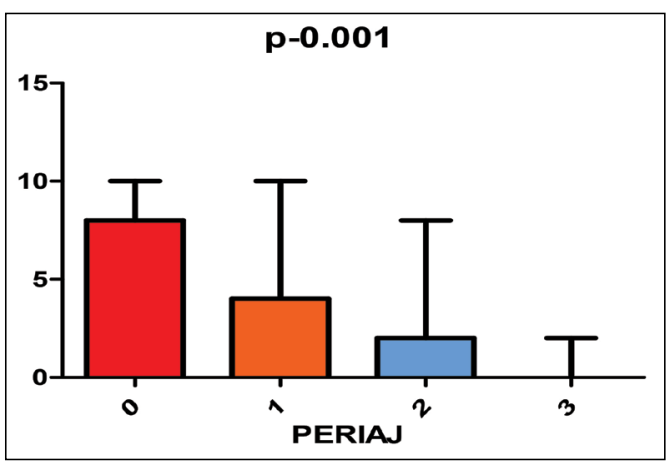

FIGURE 10. Median age / 3-5 min. tooth brushing

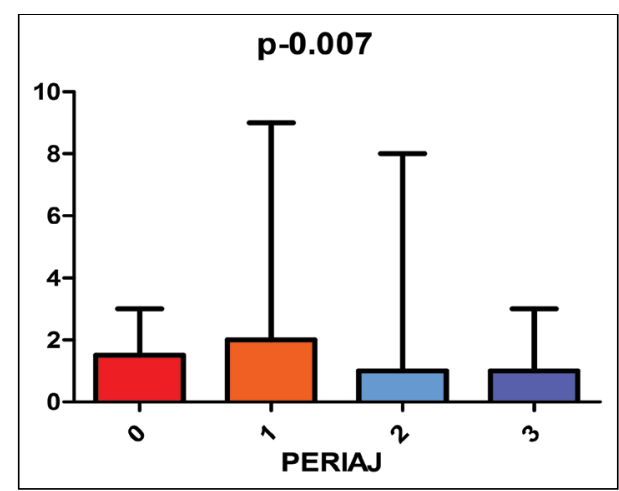

FIGURE 11. Median age / 5-10 min. tooth brushing

\section{Do you use mouthwash?}

a. Once a day

b. Twice a day

c. Never

To this question, the three variants of answer have been reported in equal proportions, $36 \%$ for twice a day and $31 \%$ for once a day (figure 12).

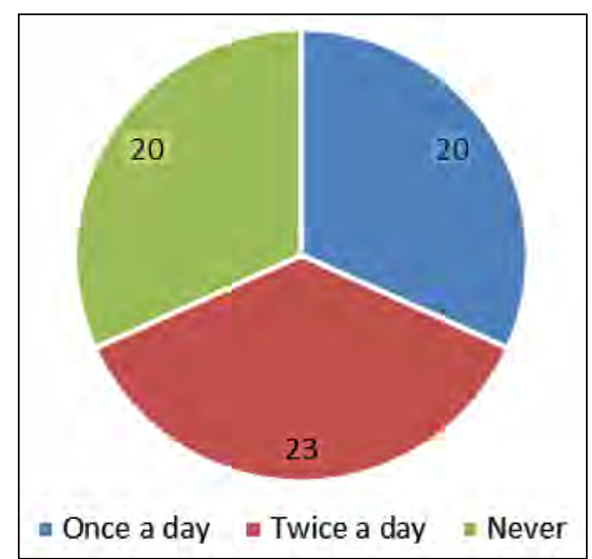

FIGURE 12. Use of mouthwash 
10. How fast did you get used to the orthodontic appliance?
a. very fast
b. relatively fast
c. very hard

Most respondents, 52\%, specified that they got used fast / very fast to the mobile orthodontic appliance, so the adjustment to a new situation was not a difficult one (figure 13).

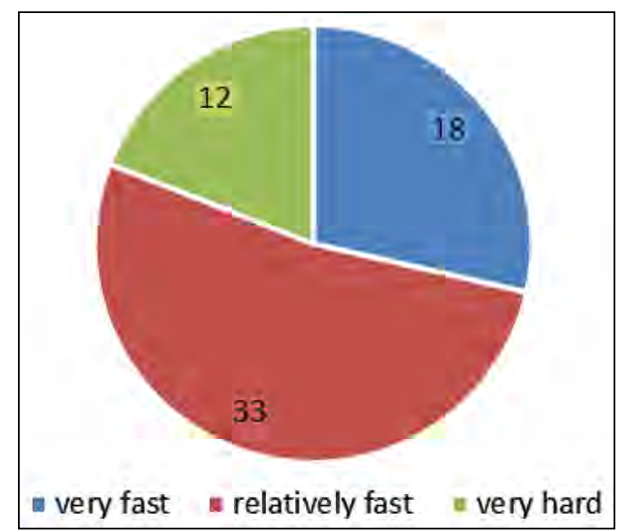

FIGURE 13. Adjustment to the mobile appliance

11. Do you consider important to use an appliance?

a. very important

b. less important

c. I don't know, my parents brought me

Although the patients are children, the great majority of the respondents $82 \%$ were aware of the importance of using the orthodontic appliance (figure 14).

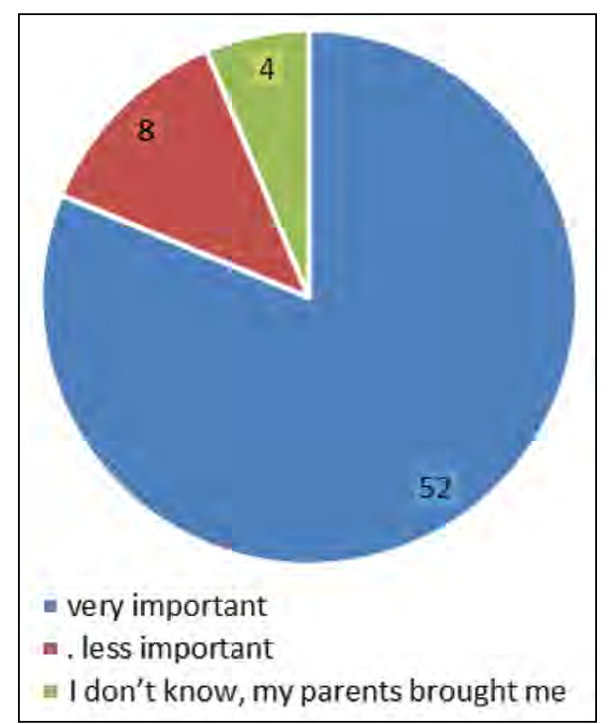

FIGURE 14. The importance of the orthodontic appliance

\section{What bad habits do you have?}

a. finger sucking

b. onychophagia (nail biting)

c. mouth breathing

d. infantile deglutition (tongue thrust swallowing)

This question had also some psychological implications, and the great majority have not ticked answers, resulting in the fact that bad habits are not acknowledged by the patients (figure 15). The thumb sucking was reported in $42 \%$ of cases, following by oral respiration in $22 \%$ of cases.

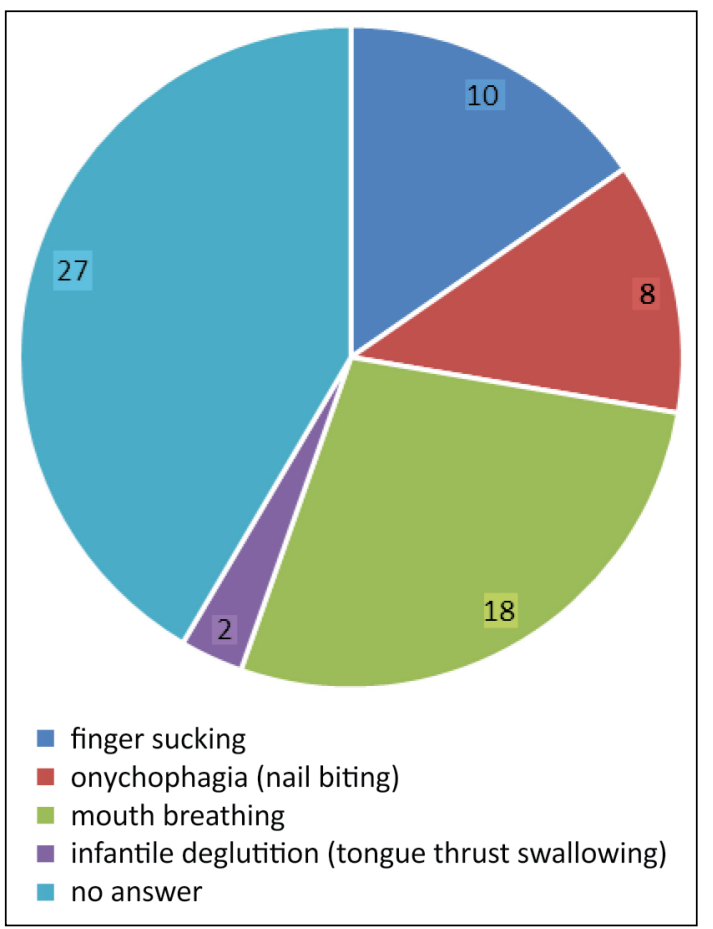

FIGURE 15. Bad habits

13. What is the impact of the orthodontic appliance on school activity?

a. I relate better with my classmates

b. I am part of the envied group

c. classmates avoid me (they make jokes)

To this question, more than $50 \%$ of the total respondents have affirmed that they relate well with their classmates after the orthodontic appliance has been applied (figure 16). It is extremely important the acceptance in the circle of friends of children with small shortcomings: dental-maxillary anomalies, vision and hearing disorders, fact that encourages them to continue the treatment. 


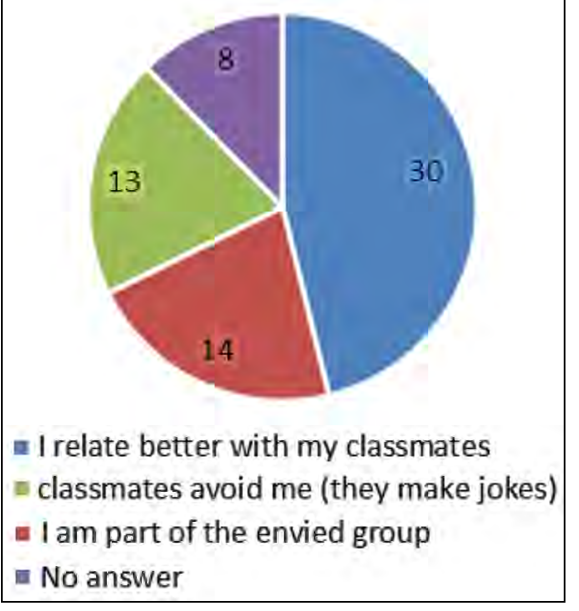

FIGURE 16. Friends perception of the orthodontic appliance

\section{Which do you think is the major benefit of using an orthodontic appliance?}

a. a more beautiful smile, healthier teeth

b. improves chewing

c. increase of self-esteem

d. giving up bad habits

The answers to the last question of the questionnaire indicate that a high percentage of the patients $80 \%$ are aware that by following the orthodontic treatment will benefit them an improved esthetic aspect and healthy teeth (figure 17).

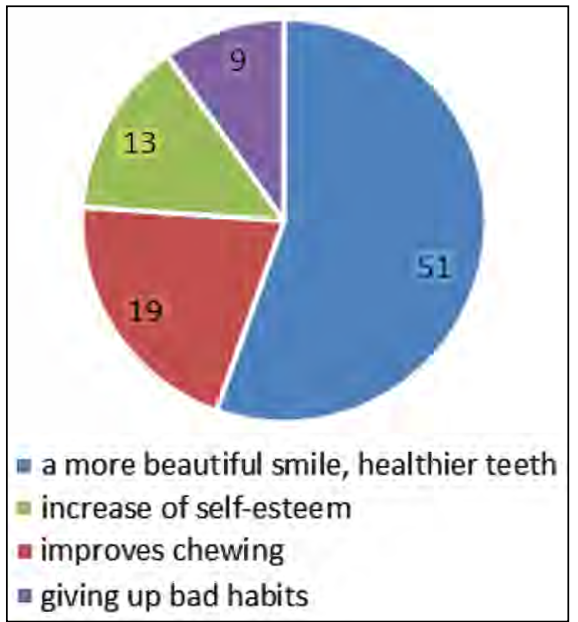

FIGURE 17. Benefits of the orthodontic treatment

\section{DISCUSSIONS}

There are a lot of studies in the specialized literature that tried to establish correlations between the way of assimilation and perception of orthodontic appliances and the dental-maxillary state, by analyzing the cavities indicators, and the manner in which the fixed or mobile orthodontic appliances have changed the dietary habits, and dental hygiene and perception on esthetics. Our study is a first feedback of the perception of patients included in the of Dental-Maxillary Anomalies Prevention Program initiated and sponsored by Bucharest City Hall, called Mobile-Smile, for school children with ages between 7 and 16 years.

The use of adjuvant means of oral hygiene and of electric toothbrushes may lead to an increase in the tooth brushing time in relation to the allotted time before the beginning of the treatment (4). The patients questioned by us have noticed a change related to the oral hygiene habits. Most of them have noticed an increase of the time allotted to tooth brushing, as well as in its frequency. The female patients from our study paid closer attention to their oral hygiene $(p=0.0044)$, performing a tooth brushing 3 times or more in a day (61\%), compared to the male patients, who predominantly brush their teeth twice a day (55\%).

Multiple studies compare the efficiency of the manual toothbrush to the electric toothbrush, obtaining mixed results. Only a single study demonstrates a greater efficiency of the manual toothbrush in removing the dental plaque compared to the electric toothbrush $(5,6)$. Other authors, on the other hand, have not found any difference between the effectiveness of the two types of toothbrushes $(7,8)$. Among the patients included in our study, $58.59 \%$ use the manual toothbrush as main means of oral hygiene. The electric toothbrush is less used $(24.44 \%)$, and an even less number of patients use alternatively both types of toothbrushes (18\%). Dental floss (super floss) is the least used auxiliary means of dental hygiene.

A similar study, developed in India on a batch of 40 patients with ages between 18 and 30 years, shows that only $2 \%$ of the patients use dental floss, but $7 \%$ of them use interdental brushes, $42 \%$ of them use mouthwash, $24 \%$ of them once a day and $18 \%$ twice a day (9). Still in India, Mayuresh J Baheti and collaborators based on a study performed on a batch of 150 patients, most of them with ages between 13 and $20(3,5)$ have noticed the use of interdental brush in a proportion of $22.60 \%$, and of mouthwash in a proportion of $31.33 \%$. Similar results have been obtained in a study managed by Priscila Ariede and collaborators in Brasil, where 
only $11.11 \%$ among the 27 volunteers included in the study use dental floss and interdental brushes $(6,10)$.

The results of this study may be regarded with certain limitations, such as the low number of the batch of study and the specific population, schoolchildren from Bucharest. Although the specific population may make difficult to generalize the results to other geographic regions, this study contributes to present a positive feedback among the children patients, wearers of mobile appliances, that were easily accepted and contribute to the improvement of the esthetic aspect and increase of self-esteem.

\section{CONCLUSIONS}

1. The assessment of the patient's perception on the role of the mobile orthodontic appliance is an effective method, relatively simple of quantifying the oral-dental changes and general ones induced by the orthodontic therapy.

2. The answers of the survey fall under a normal feedback of patients, users of orthodontic

\section{REFERENCES}

1. Trimpeneers LM et al. Effect of electric toothbrushes versus manual toothbrushes on removal of plaque and periodontal status during orthodontic treatment. Am J Orthod Dentofacial Orthop. 1997. 111:492-497.

2. Heintze S D, Jost-Brinkmann PG, Loundos J. Effectiveness of three different types of electric toothbrushes compared with a manual technique in orthodontic patients. Am J Orthod Dentofacial Orthop. 1996 Dec;110(6):630-8.

3. Borutta A, Pala E, Fischer T. Effectiveness of a powered toothbrush compared with a manual toothbrush for orthodontic patients with orthodontic appliances. J Clin Dent. 2002;13(4):131-7.

4. Vijayendra Pandey et al. Impact of dental neglect score on oral health among patients receiving orthodontic treatment: A crosssectional study. J Int Soc Prev Community Dent. 2016 Mar-Apr; 6(2): 120-124.

5. Mayuresh J Baheti et al. Survey on oral hygiene protocols among orthodontic correction-seeking individuals. Journal of Education and Ethics in Dentistry. 2015;5(1);8-13. appliances, who besides the direct effect of correcting the anomaly, have reported also an improvement of their dental hygiene and an increase of the frequency and duration of tooth brushing (3-5 min.).

3. A high percentage of respondents (over $80 \%$ ) have confirmed the importance of the orthodontic treatment, mentioning that they have got used to it relatively fast and relate well with their classmates.

4. There is an obvious tendency of increased desire for an orthodontic therapy within the analyzed population, most of the patients being aware of the role and need to wear an orthodontic appliance.

5. It is important that dentists raise awareness in the parents on the importance for children to learn as early as possible in childhood the correct measures of oral and dental hygiene, and the role of periodic check-ups in order to detect bad habits and incipient dental-maxillary anomalies, ensuring this way a harmonious development of children and increasing the quality of life.

Conflict of interest: none declared Financial support: none declared

6. Priscila Ariede, Petinuci Bardal et al. Education and motivation in oral health - preventing disease and promoting health in patients undergoing orthodontic treatment. Dental Press J. Orthod. 2011. vol.16 (3).

7. Azaripour A. et al. Braces versus Invisalign: Gingival parameters and patients' satisfaction during treatment: A cross-sectional study. BMC Oral Health 2015;15.

8. Kvam E et al. Traumatic ulcers and pain in adults during orthodontic treatment.1989. Community Dentistry and Oral Epidemiology. 1989; 17(3):154-7.

9. Jürgensen N, Petersen PE. Oral health and the impact of sociobehavioural factors in a cross sectional survey of 12-year old schoolchildren in Laos. BMC Oral Health. 2009; 9:29.

10. Broder HL, Slade G, Caine R et al. Perceived impact of oral health conditions and quality of life among American adolescents. J Dent Res. 2017;26(8):201-205. 